\title{
Zero-field superfluid density in a $d$-wave superconductor evaluated from muon-spin-rotation experiments in the vortex state
}

\author{
R. Khasanov, ${ }^{1, *}$ Takeshi Kondo, ${ }^{2,3}$ S. Strässle, ${ }^{4}$ D. O. G. Heron, ${ }^{5}$ A. Kaminski, ${ }^{2}$ H. Keller, ${ }^{4}$ \\ S. L. Lee, ${ }^{5}$ and Tsunehiro Takeuchi ${ }^{3,6}$ \\ ${ }^{1}$ Laboratory for Muon Spin Spectroscopy, Paul Scherrer Institut, CH-5232 Villigen PSI, Switzerland \\ ${ }^{2}$ Department of Physics and Astronomy and Ames Laboratory, Iowa State University, Ames, Iowa 50011, USA \\ ${ }^{3}$ Department of Crystalline Materials Science, Nagoya University, Nagoya 464-8603, Japan \\ ${ }^{4}$ Physik-Institut der Universität Zürich, Winterthurerstrasse 190, CH-8057 Zürich, Switzerland \\ ${ }^{5}$ School of Physics and Astronomy, University of St. Andrews, Fife KY16 9SS, United Kingdom \\ ${ }^{6}$ EcoTopia Science Institute, Nagoya University, Nagoya 464-8603, Japan \\ (Received 13 November 2008; revised manuscript received 15 March 2009; published 14 May 2009)
}

\begin{abstract}
We present an approach that allows the reconstruction of the zero-field magnetic penetration depth $\lambda_{0}$ based on the results of muon-spin-rotation $(\mu \mathrm{SR})$ experiments conducted in a superconductor in the vortex state. It was successfully applied to describe the $\mu \mathrm{SR}$ experiments in optimally doped $(\mathrm{BiPb})_{2}(\mathrm{SrLa})_{2} \mathrm{CuO}_{6+\delta}(\mathrm{OP}$ Bi2201). We found that in unconventional $d$-wave superconductors (such as OP Bi2201) only at relatively low magnetic fields $\left[B / B_{c 2} \leq 10^{-3} ; B_{c 2}\right.$ is the upper critical field $]$ the effective penetration depth $\lambda_{\text {eff }}$, obtained in $\mu$ SR experiment, is a good measure of $\lambda_{0}$. The high-field data need to be evaluated accounting for both the nonlinear and the nonlocal corrections.
\end{abstract}

DOI: 10.1103/PhysRevB.79.180507

PACS number(s): 74.72.Hs, 74.25.Jb, 76.75.+i

Muon-spin-rotation ( $\mu \mathrm{SR})$ measurements in the vortex state of type-II superconductors provide valuable information on the superconducting properties. An important advantage of this method is that the muons probe the bulk of the material, and the results are not complicated by surface imperfections. The quantitative parameters extracted from $\mu \mathrm{SR}$ experiments depend, however, on the details of the model used to reconstruct the internal magnetic field distribution in the superconductor in the vortex state. So far, field distributions measured by means of $\mu \mathrm{SR}$ were analyzed within the framework of analytical models based on London and Ginzburg-Landau (GL) theories, which can be applied, in general, to conventional superconductors with a single isotropic energy gap. ${ }^{1-3}$ The situation becomes much more complicated in the case of unconventional superconductors, such as cuprates and $\mathrm{MgB}_{2}$. It was found, that the effective magnetic field penetration depth $\lambda_{\text {eff }}$ extracted from $\mu$ SR measurements depends on the applied magnetic field (see, e.g., Refs. 4-11) which is unexpected within the GL theory. Here $\lambda_{\text {eff }}$ refers to a quantity evaluated from $\mu \mathrm{SR}$ experiments conducted in a superconductor in the vortex state $\left(H>H_{c 1}\right.$; $H_{c 1}$ denotes the lower critical field) in contrast to the zerofield penetration depth $\lambda_{0}$ as obtained from Meissner state experiments $\left(H \ll H_{c 1}\right)$. In addition, it was observed that not only the absolute value but also the shape of $\lambda_{\text {eff }}^{-2}(T)$ change with field. ${ }^{5,7,9-11}$ In this respect the question concerning the relation of $\lambda_{\text {eff }}$ to $\lambda_{0}$, which is generally assumed to be proportional to the superfluid density $\left(\lambda_{0}^{-2} \propto \rho_{s}\right)$, becomes very important.

Here we present an approach that allows the reconstruction of $\lambda_{0}$ based on the results of $\mu \mathrm{SR}$ experiments conducted in a superconductor in the vortex state. It was applied to describe the $\mu \mathrm{SR}$ data for the optimally doped cuprate superconductor $(\mathrm{BiPb})_{2}(\mathrm{SrLa})_{2} \mathrm{CuO}_{6+\delta} \quad(\mathrm{OP} \quad \mathrm{Bi} 2201)$. $\lambda_{\text {eff }}(T, H)$ was obtained from the measured temperature dependence of the $\mu$ SR linewidth by using numerical calcula- tions of Brandt. $^{3} \quad \lambda_{0}^{-2}(T)$ was further evaluated from $\lambda_{\text {eff }}(T, H)$ by considering the nonlinear and the nonlocal response of a superconductor with nodes in the energy gap to the applied magnetic field.

Details on the sample preparation of OP Bi2201 single crystals can be found elsewhere. ${ }^{12,13}$ Field-cooled magnetization $\left(M_{\mathrm{FC}}\right)$ measurements of OP Bi2201 were performed with a superconducting quantum interference device (SQUID) magnetometer at $\mu_{0} H=1 \mathrm{mT} \| c$ for temperatures ranging from 5 to $50 \mathrm{~K}$. The transition temperature $T_{c} \simeq 34.8 \mathrm{~K}$ was obtained as the intersect of the linearly extrapolated $M_{\mathrm{FC}}(T)$ curve with the $M=0$ line [see Fig. 1(a)].

The transverse-field $\mu$ SR experiments were carried out at the $\pi \mathrm{M} 3$ beam line at the Paul Scherrer Institute (Villigen, Switzerland). Two OP Bi2201 single crystals $\left(4 \times 2 \times 0.1 \mathrm{~mm}^{3}\right)$ were mounted on a holder designed to perform $\mu \mathrm{SR}$ experiments on thin single crystalline samples.
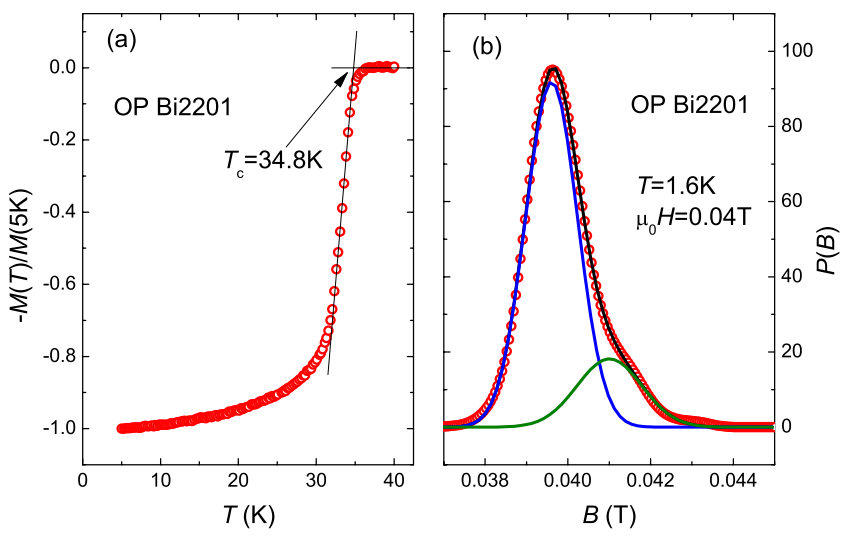

FIG. 1. (Color online) (a) Field-cooled magnetization $M_{\mathrm{FC}}(T)$ of OP Bi2201 at $\mu_{0} H=1 \mathrm{mT} \| c$. (b) The magnetic field distribution $P(B)$ of $\mathrm{OP} \mathrm{Bi} 2201$ at $T=1.6 \mathrm{~K}$ and $\mu_{0} H=0.04 \mathrm{~T}$. The lines are the best fit within a two-Gaussian approach. 


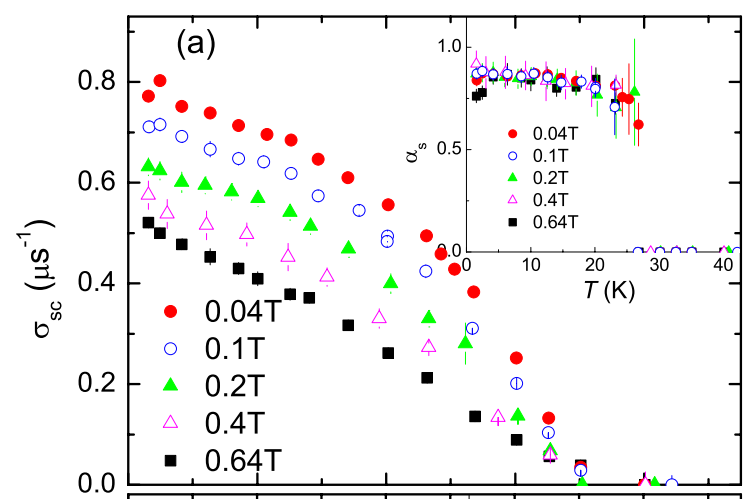

(b)

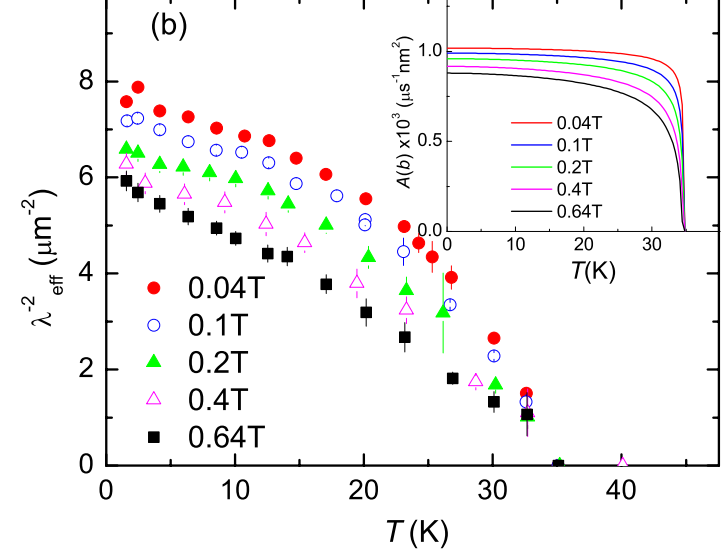

FIG. 2. (Color online) (a) Temperature dependence of $\sigma_{\mathrm{sc}} \propto \lambda_{a b}^{-2}$ of OP Bi2201 measured at $\mu_{0} H=0.04,0.1,0.2,0.4$, and $0.64 \mathrm{~T}$. The inset shows the dependence of the skewness parameter $\alpha_{s}$ on $T$. (b) Temperature dependence of the effective magnetic penetration depth $\lambda_{\text {eff }}^{-2}$ of OP Bi2201 reconstructed from $\sigma_{\text {sc }}(T)$ measured at $\mu_{0} H=0.04,0.1,0.2,0.4$, and $0.64 \mathrm{~T}$. The inset shows the temperature dependence of the correction factor $A(b)=\sigma_{\mathrm{sc}} \cdot \lambda_{\mathrm{eff}}^{2}$.

The sample was field cooled from above $T_{c}$ to $1.6 \mathrm{~K}$ in series of fields ranging from $5 \mathrm{mT}$ to $0.64 \mathrm{~T}$. The magnetic field was applied parallel to the $c$ axis and transverse to the muonspin polarization. The typical counting statistics were $\sim 15-18 \times 10^{6}$ muon detections per data point. The experimental data were analyzed using the procedure described in Refs. 9, 11, and 14. The analysis is based on a twocomponent Gaussian fit of the $\mu$ SR time spectra which allows to describe the asymmetric local magnetic field distribution $P(B)$ in the superconductor in the vortex state [see Fig. 1(b)]. The superconducting part of the square root of the second moment $\left(\sigma_{\mathrm{sc}} \propto \lambda^{-2}\right)$ was obtained by subtracting the normal state nuclear moment contribution $\left(\sigma_{\mathrm{nm}}\right)$ from the measured second moment of $P(B)\left(\sigma^{2}\right)$, as $\sigma_{\mathrm{sc}}^{2}=\sigma^{2}-\sigma_{\mathrm{nm}}^{2} \cdot{ }^{9}$ Since the magnetic field was applied along the crystallographic $c$ axis, our experiments provide direct information on the in-plane magnetic penetration depth $\lambda_{a b}$.

The temperature dependences of $\sigma_{\mathrm{sc}} \propto \lambda_{a b}^{-2}$ measured in $\mu_{0} H=0.04,0.1,0.2,0.4$, and $0.64 \mathrm{~T}$ are shown in Fig. 2(a). To ensure that $\sigma_{\mathrm{sc}}(T)$ is determined primarily by the variance in the magnetic field due to the vortex lattice (VL), we plot in the inset the corresponding values of the skewness parameter $\alpha_{s}=\left\langle\Delta B^{3}\right\rangle^{1 / 3} /\left\langle\Delta B^{2}\right\rangle^{1 / 2}\left[\left\langle\Delta B^{n}\right\rangle\right.$ is the $n$th central moment of $P(B)] . \alpha_{s}$ is a dimensionless measure of the asymmetry of $P(B)$, the variation in which reflects underlying changes in the vortex structure. ${ }^{15}$ For an ideal triangular VL $\alpha_{s} \simeq 1.2$. It is very sensitive to structural changes in the VL which can occur as a function of temperature and/or magnetic field. ${ }^{15,16}$ The inset in Fig. 2(a) implies that in OP Bi2201 $\alpha_{s}(T, H)$ is almost constant for $1.6 \leq T \leq 26 \mathrm{~K}$ and smaller than the ideal value of 1.2 , which can be caused by a number of factors including microscopic field gradients due to interaction of flux expulsion with pinning, different geometric factors due to the range of crystal sizes and shapes, spatial variation in the penetration depth. ${ }^{17}$ The sharp change in $\alpha_{s}$ at $T \simeq 30 \mathrm{~K}$ is similar to what was observed in $\mathrm{Bi} 2212$, where it was attributed to VL melting. ${ }^{15,16}$ Therefore, we conclude that for temperatures $1.6<T \leqq 30 \mathrm{~K}$ the $T$ variation of $\sigma_{\mathrm{sc}}$ in OP Bi2201 studied in the present work reflects the intrinsic behavior of the in-plane magnetic penetration depth $\lambda_{a b}(T)$.

From the measured $\sigma_{\mathrm{sc}}(T, H)$ we reconstructed $\lambda_{\mathrm{eff}}^{-2}(T, H)$ by using the relation ${ }^{18}$

$$
\sigma_{\mathrm{sc}}(b)\left[\mu \mathrm{s}^{-1}\right]=A(b) \lambda_{\mathrm{eff}}^{-2}\left[\mathrm{~nm}^{-2}\right],
$$

which accounts for decrease in the field variance within the VL with increasing magnetic field. ${ }^{1,3}$ The correction factor $A(b)$ depends only on the reduced field $b=B / B_{c 2}\left(B_{c 2}\right.$ is the upper critical field). For a superconductor with a Ginzburg-Landau parameter $\kappa=\lambda / \xi \geq 5$ measured in fields ranging from $0.25 / \kappa^{1.3} \leq b \leq 1$, $A(b)$ is independent of $\kappa$ and is approximated as $A(b)=4.83 \cdot 10^{4}(1-b)\left[1+1.21(1-\sqrt{b})^{3}\right] \mu \mathrm{s}^{-1} \mathrm{~nm}^{2} .^{3}$

The reconstructed $\lambda_{\text {eff }}^{-2}(T, H=$ const $)$ curves are shown in Fig. 2(b). The corresponding $A(b)$ dependences are displayed in the inset. The calculations were made for $B_{c 2}(0)=50 \mathrm{~T} .{ }^{19}$ The temperature dependence of $B_{c 2}$ was assumed to follow the Werthamer-Helfand-Hohenberg prediction. $^{20}$ Below $T \sim 20 \mathrm{~K}, \lambda_{\text {eff }}^{-2}$ is linear in $T$, as is expected for a superconductor with nodes in the energy gap. Note that there is no pronounced upturn on $\lambda_{\text {eff }}$ as observed in other cuprates, ${ }^{9-11}$ which justifies our analysis based on purely $d$-wave symmetry of the order parameter. Figure 2(b) also implies that in the whole temperature region (from $T \simeq 1.6 \mathrm{~K}$ up to $T_{c}$ ), $\lambda_{\text {eff }}^{-2}(T, H)$ decreases with increasing field. This contrasts with the results obtained by using a similar procedure for the ternary boride $\mathrm{Li}_{2} \mathrm{Pd}_{3} \mathrm{~B}$ and the electron-doped $\mathrm{Sr}_{0.9} \mathrm{La}_{0.1} \mathrm{CuO}_{2} \cdot{ }^{18,21}$ For these two superconductors the $\lambda_{\text {eff }}^{-2}(T, H)$ curves were found to collapse onto a single curve. Since $\mathrm{Li}_{2} \mathrm{Pd}_{3} \mathrm{~B}$ and $\mathrm{Sr}_{0.9} \mathrm{La}_{0.1} \mathrm{CuO}_{2}$ are supposed to be fully gaped, ${ }^{18-25}$ we may conclude that the field dependence of $\lambda_{\text {eff }}^{-2}(T)$, shown in Fig. 2(b), is caused by the presence of nodes in the superconducting energy gap of OP Bi2201. ${ }^{26}$

As shown in Refs. 27 and 28, the magnetic field dependence of $\lambda_{\text {eff }}$ arises from the nonlocal and the nonlinear response of a superconductor with nodes in the energy gap to the applied magnetic field. The nonlinear correction to $\lambda_{a b}$ appears due to the magnetic field induced quasiparticle excitation over the gap nodes. ${ }^{29}$ According to Volovik, ${ }^{29}$ the density of the delocalized states increases proportionally to $\sqrt{b}$. The nonlocal correction to $\lambda_{a b}$ appears from the response of electrons with momenta on the Fermi surface close to the gap nodes. This is because the coherence length $\xi$, being inversely proportional to the gap, becomes very large close to the nodes and, formally, diverges at the nodal points. 


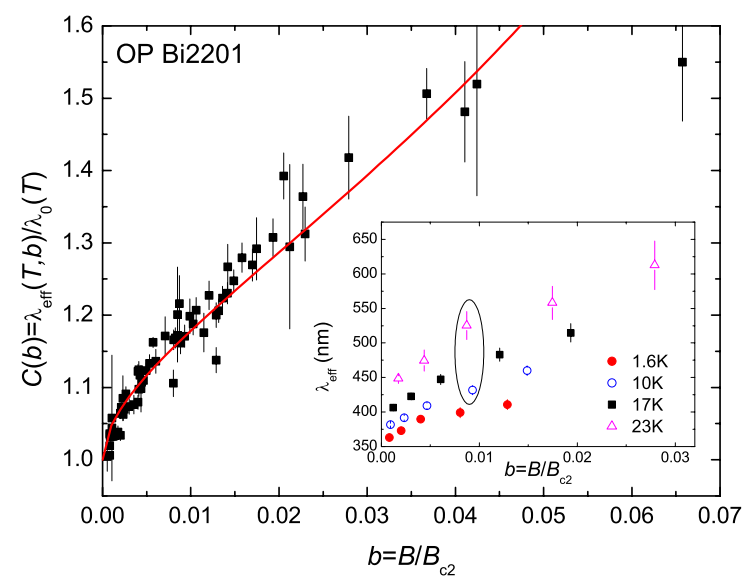

FIG. 3. (Color online) Dependence of the proportionality factor $C(b)=\lambda_{\text {eff }}(T, b) / \lambda_{0}(T)$ on the reduced field $b=B / B_{c 2}$ of OP Bi2201. The solid line is the fit by means of Eq. (3). The inset shows the dependence of $\lambda_{\text {eff }}$ at $T=1.6,10,17$, and $23 \mathrm{~K}$ on the reduced field $b=B / B_{c 2}$.

Thus, there exist areas on the Fermi surface where $\lambda / \xi \lesssim 1$, and the response of a superconductor to an applied magnetic field becomes highly nonlocal. ${ }^{28}$

In order to reconstruct the temperature dependence of the superfluid density $\rho_{s} \propto \lambda_{0}^{-2}$ we considered that the proportionality factor relating $\lambda_{\text {eff }}$ to $\lambda_{0}$ is a function of the reduced magnetic field $b$ only so that

$$
\lambda_{\mathrm{eff}}(b, T)=C(b) \lambda_{0}(T) .
$$

This statement is correct, at least, in the case of nonlinear corrections which scale with $\sqrt{b} .^{29-31}$

The fact that the field and the temperature dependences of $\lambda_{\text {eff }}$ are described by separate terms in Eq. (2) allows reconstruction of $\lambda_{0}(T)$. In order to demonstrate this, we refer to the inset in Fig. 3 which shows the dependence of $\lambda_{\text {eff }}$ on the magnetic field for some selected temperatures. The reduced field $b$ for $\lambda_{\text {eff }}$ measured at $T=10 \mathrm{~K}$ and $\mu_{0} H=0.4 \mathrm{~T}$ (lower point in the oval selection) is almost the same as the one for the point at $T=23 \mathrm{~K}$ and $\mu_{0} H=0.2 \mathrm{~T}$ (upper point). This implies that coefficients $C(b)$ for these two points are nearly the same and that the difference in the absolute values of $\lambda_{\text {eff }}(10 \mathrm{~K}, 0.4 \mathrm{~T})$ and $\lambda_{\text {eff }}(23 \mathrm{~K}, 0.2 \mathrm{~T})$ is due to different values of $\lambda_{0}$. The reconstruction procedure was performed in the following way. First, from $\lambda_{\text {eff }}^{-2}(T, H)$ plotted in Fig. 2(b), $\lambda_{\text {eff }}(b)$ was reconstructed for various constant temperatures (see inset in Fig. 3). Second, the resulting $\lambda_{\text {eff }}(T=$ const, $b$ ) curves were scaled in order to have them collapsing on a single curve (see Fig. 3). According to Eq. (2) this curve corresponds to $C(b)=\lambda_{\text {eff }}(T, b) / \lambda_{0}(T)$, while the scaling factor, in turn, corresponds to $\lambda_{0}(T)$ (in order to get $\lambda_{0}$ one should take into account that $\lambda_{\text {eff }} \rightarrow \lambda_{0}$ for $b \rightarrow 0$ ). The solid line represents the result of the fit using

$$
\lambda_{\text {eff }}(b) / \lambda_{0}=C(b)=(1-K \sqrt{b})^{-1 / 2},
$$

which takes into account the nonlinear correction to $\lambda_{0}$ for a superconductor with $d$-wave energy gap. ${ }^{6,31}$ Here the parameter $K$ depends on the strength of the nonlinear effect. It is

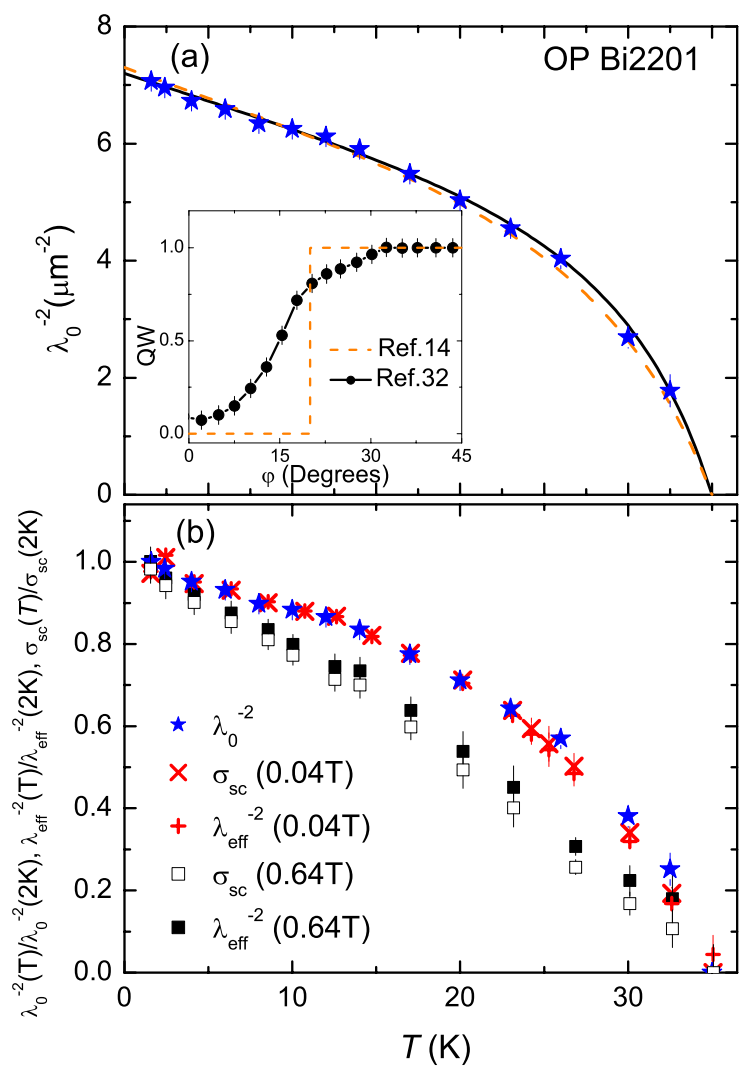

FIG. 4. (Color online) (a) Temperature dependence of $\lambda_{0}^{-2}$ of OP Bi2201 reconstructed from $\lambda_{\text {eff }}^{-2}(T, H)$ measured at $\mu_{0} H=0.04,0.1$, $0.2,0.4$, and $0.64 \mathrm{~T}$ [see Fig. 2(b)]. The solid lines correspond to the analysis with $\operatorname{QW}(\varphi)$ functions shown in the inset. See text for details. (b) Temperature dependence of the normalized $\lambda_{0}^{-2}$, $\lambda_{\text {eff }}^{-2}(0.04 \mathrm{~T}), \sigma_{\mathrm{sc}}(0.04 \mathrm{~T}), \lambda_{\mathrm{eff}}^{-2}(0.64 \mathrm{~T})$, and $\sigma_{\mathrm{sc}}(0.64 \mathrm{~T})$.

obvious that the "nonlinear" curve describes the experimental $C(b)=\lambda_{\text {eff }}(b) / \lambda_{0}$ dependence reasonably well. In particular, it reproduces the curvature at $b \leqq 0.01$ and the linear increase in $C(b)$ for $0.01 \leqq b \leqq 0.05$. We believe, however, that the whole $\lambda_{\text {eff }}(b) / \lambda_{0}$ curve must be a combination of both nonlinear and nonlocal correction effects, similar to the results of Ref. 27.

The temperature dependence of $\lambda_{0}^{-2}$ and the comparison of $\lambda_{0}^{-2}(T)$ with $\sigma_{\mathrm{sc}}(T)$ and $\lambda_{\text {eff }}^{-2}(T)$ measured at $\mu_{0} H=0.04$ and $0.64 \mathrm{~T}$ are presented in Fig. 4. Both $\sigma_{\mathrm{sc}}(T)$ and $\lambda_{\mathrm{eff}}^{-2}(T)$ measured at $\mu_{0} H=0.04 \mathrm{~T}$ almost coincide with each other as well as with $\lambda_{0}^{-2}(T)$. This implies that the two sets of corrections, namely, the first, accounting for decrease in the second moment of the $\mu \mathrm{SR}$ line with increasing field [Eq. (1) and the inset in Fig. 2(b)], and the second, arising due to the nonlocal and the nonlinear response of a superconductor with nodes in the gap to the applied magnetic field [Eq. (2) and Fig. 3], are negligible at relatively low fields. Consequently, $\sigma_{\mathrm{sc}}$ measured at $\mu_{0} H=0.04 \mathrm{~T}$ is still a good measure of the zero-field superfluid density $\rho_{s} \propto \lambda_{0}^{-2}$. On the other hand, $\sigma_{\text {sc }}(T)$ and $\lambda_{\text {eff }}^{-2}(T)$ for $\mu_{0} H=0.64$ T differ substantially from each other and from the resulting $\lambda_{0}^{-2}(T)$. This implies that for high fields both above-mentioned corrections have to be taken into account.

The resulting $\lambda_{0}^{-2}(T)$ dependence was analyzed within the same scheme as described in Ref. 14, 


$$
\frac{\lambda^{-2}(T)}{\lambda^{-2}(0)}=1+\frac{2}{S_{\mathrm{QW}}} \int_{0}^{\pi / 4} \int_{\Delta(T, \varphi)}^{\infty}\left(\frac{\partial f}{\partial E}\right) \frac{\mathrm{QW}(\varphi) E d E d \varphi}{\sqrt{E^{2}-\Delta(T, \varphi)^{2}}}
$$

Here $f=\left[1+\exp \left(E / k_{B} T\right)\right]^{-1}$ denotes the Fermi function, $\varphi$ is the angle along the Fermi surface $(\varphi=0$ and $\pi / 4$ correspond to the antinodal and the nodal points, respectively), $\Delta(T, \varphi)$ $=\Delta_{0} \tanh \left\{1.82\left[1.018\left(T_{c} / T-1\right)^{0.51}\right]\right\} \cos (2 \varphi)$ is the assumed $d$-wave superconducting energy gap $\left(\Delta_{0}\right.$ is the maximum gap value at $T=0), \mathrm{QW}(\varphi)$ accounts for the relative weight of the quasiparticles condensed into the Cooper pairs as a function of $\varphi$, and $S_{\mathrm{QW}}=\int_{0}^{\pi / 4} \mathrm{QW}(\varphi) d \varphi$. The dashed and solid lines in Fig. 4(a) correspond to the case when: (i) $\mathrm{QW}(\varphi)$ changes abruptly from $\mathrm{QW}(\varphi)=0$ to 1 at $\varphi=\varphi_{0} \simeq 20^{\circ}$ and $\Delta_{0}$ $=15 \mathrm{meV},{ }^{14}$ and (ii) $\mathrm{QW}(\varphi)$ follows the dependence obtained for OP Bi2201 in recent angle resolved photoemission spectroscopy (ARPES) experiments, ${ }^{32}$ and $\Delta_{0}=13.7 \mathrm{meV}$. The corresponding $\mathrm{QW}(\varphi)$ dependences are shown in the inset. Obviously, both theoretical curves describe the experimental data reasonably well, thus confirming the validity of the previously used approach, ${ }^{14}$ as well as consistency of
$\lambda_{0}^{-2}(T)$ with $\mathrm{QW}(\varphi)$ reported in Ref. 32. Note that in the latter case the absolute value of the gap $\Delta_{0}=13.7 \mathrm{meV}$ is smaller than $\Delta_{0} \simeq 18 \mathrm{meV}$ reported in Ref. 32 .

To summarize, $\mu \mathrm{SR}$ measurements were performed on single crystals of the optimally doped cuprate superconductor $(\mathrm{BiPb})_{2}(\mathrm{SrLa})_{2} \mathrm{CuO}_{6+\delta}$. It was demonstrated that in unconventional $d$-wave superconductors (such as OP Bi2201) $\mu$ SR experiments taken in various magnetic fields allow a reliable evaluation of the zero-field superfluid density $\rho_{s} \propto \lambda_{0}^{-2}$. Our results imply that only at relatively low magnetic fields $\left[b \leqslant 10^{-3}\right] \lambda_{\text {eff }}(T)$, obtained in $\mu$ SR experiment, is a good measure of $\lambda_{0}(T)$. The high-field $\mu \mathrm{SR}$ data need to be evaluated by taking into account both nonlinear and nonlocal corrections.

This work was performed at the Swiss Muon Source $(\mathrm{S} \mu \mathrm{S})$, Paul Scherrer Institute (PSI, Switzerland). Work at the Ames Laboratory was supported by the Department of Energy, Basic Energy Sciences under Contract No. DEAC02-07CH11358. The financial support of the Swiss National Foundation (SNF) is gratefully acknowledged.
*Corresponding author. rustem.khasanov@psi.ch

${ }^{1}$ E. H. Brandt, Phys. Rev. B 37, 2349 (1988).

${ }^{2}$ A. Yaouanc, P. Dalmas de Réotier, and E. H. Brandt, Phys. Rev. B 55, 11107 (1997).

${ }^{3}$ E. H. Brandt, Phys. Rev. B 68, 054506 (2003).

${ }^{4}$ J. E. Sonier, J. H. Brewer, and R. F. Kiefl, Rev. Mod. Phys. 72, 769 (2000)

${ }^{5}$ J. E. Sonier, J. H. Brewer, R. F. Kiefl, G. D. Morris, R. I. Miller, D. A. Bonn, J. Chakhalian, R. H. Heffner, W. N. Hardy, and R. Liang, Phys. Rev. Lett. 83, 4156 (1999).

${ }^{6}$ R. Kadono, J. Phys.: Condens. Matter 16, S4421 (2004).

${ }^{7}$ C. Niedermayer, C. Bernhard, T. Holden, R. K. Kremer, and K. Ahn, Phys. Rev. B 65, 094512 (2002).

${ }^{8}$ S. Serventi, G. Allodi, R. De Renzi, G. Guidi, L. Romano, P. Manfrinetti, A. Palenzona, C. Niedermayer, A. Amato, and C. Baines, Phys. Rev. Lett. 93, 217003 (2004).

${ }^{9}$ R. Khasanov, A. Shengelaya, A. Maisuradze, F. La Mattina, A. Bussmann-Holder, H. Keller, and K. A. Müller, Phys. Rev. Lett. 98, 057007 (2007).

${ }^{10}$ R. Khasanov, S. Strässle, D. Di Castro, T. Masui, S. Miyasaka, S. Tajima, A. Bussmann-Holder, and H. Keller, Phys. Rev. Lett. 99, 237601 (2007).

${ }^{11}$ R. Khasanov et al., J. Supercond. Novel Magn. 21, 81 (2008).

${ }^{12}$ T. Kondo et al., J. Electron Spectrosc. Relat. Phenom. 137-140, 663 (2004)

${ }^{13}$ T. Kondo, T. Takeuchi, U. Mizutani, T. Yokoya, S. Tsuda, and S. Shin, Phys. Rev. B 72, 024533 (2005).

${ }^{14}$ R. Khasanov, T. Kondo, S. Strässle, D. O. G. Heron, A. Kaminski, H. Keller, S. L. Lee, and T. Takeuchi, Phys. Rev. Lett. 101, 227002 (2008).

${ }^{15}$ S. L. Lee et al., Phys. Rev. Lett. 71, 3862 (1993).
${ }^{16}$ C. M. Aegerter, J. Hofer, I. M. Savic, H. Keller, S. L. Lee, C. Ager, S. H. Lloyd, and E. M. Forgan, Phys. Rev. B 57, 1253 (1998).

${ }^{17}$ T. M. Riseman et al., Phys. Rev. B 52, 10569 (1995).

${ }^{18}$ R. Khasanov, A. Shengelaya, A. Maisuradze, D. Di Castro, I. M. Savic, S. Weyeneth, M. S. Park, D. J. Jang, S. I. Lee, and H. Keller, Phys. Rev. B 77, 184512 (2008).

${ }^{19}$ Y. Wang et al., Science 299, 86 (2003).

${ }^{20}$ N. R. Werthamer, E. Helfand, and P. C. Hohenberg, Phys. Rev. 147, 295 (1966).

${ }^{21}$ R. Khasanov, I. L. Landau, C. Baines, F. La Mattina, A. Maisuradze, K. Togano, and H. Keller, Phys. Rev. B 73, 214528 (2006).

${ }^{22}$ C. T. Chen, P. Seneor, N. C. Yeh, R. P. Vasquez, L. D. Bell, C. U. Jung, J. Y. Kim, M. S. Park, H. J. Kim, and S. I. Lee, Phys. Rev. Lett. 88, 227002 (2002).

${ }^{23}$ J. S. White et al., J. Phys.: Condens. Matter 20, 104237 (2008).

${ }^{24}$ Z. Y. Liu et al., Europhys. Lett. 69, 263 (2005).

${ }^{25}$ P. S. Häfliger et al., J. Supercond. Nov. Magn. 22, 337 (2009).

${ }^{26}$ T. Kondo, T. Takeuchi, A. Kaminski, S. Tsuda, and S. Shin, Phys. Rev. Lett. 98, 267004 (2007).

${ }^{27}$ M. H. S. Amin, M. Franz, and I. Affleck, Phys. Rev. Lett. 84, 5864 (2000).

${ }^{28}$ M. H. S. Amin, Ph.D. thesis, University of British Colombia, 1999; arXiv:cond-mat/0011455 (unpublished).

${ }^{29}$ G. E. Volovik, Pis'ma Zh. Eksp. Teor. Fiz. 58, 457 (1993) [JETP Lett. 58, 469 (1993)].

${ }^{30}$ H. Won and K. Maki, Europhys. Lett. 54, 248 (2001).

${ }^{31}$ I. Vekhter, J. P. Carbotte, and E. J. Nicol, Phys. Rev. B 59, 1417 (1999).

${ }^{32}$ T. Kondo et al., Nature (London) 457, 296 (2009). 